\title{
Reversible Leukoencephalopathy Due to Chronic Unintentional Exposure to Toluene
}

\author{
Salah U. Qureshi, Adam R. Blanchette, Ali Jawaid, Paul E. Schul,
}

Can. J. Neurol. Sci. 2009; 36: 388-389

A 40-year-old Caucasian chemical salesman was stopped by a police officer for driving erratically. He was suspected of "driving while intoxicated". Although he was confused and appeared to be intoxicated, several breathalyzer tests were negative for alcohol. The police then took him to a local hospital for an evaluation.

In the emergency room, he was disoriented and ataxic. His vitals were normal and the rest of his physical examination was unremarkable. A brain magnetic resonance image (MRI) without contrast showed extensive, diffuse white matter changes. There were increased T2-weighted signal intensity throughout the subcortical and periventricular white matter (Figure 1 and 2).

Over the next three days, the patient's mental status gradually improved; however, he remained anxious and easily distractible. His mental status examination continued to show deficits in attention and executive function. The patient was started on Quetiapine, 75mgs daily, for behavioral control.

A comprehensive diagnostic workup for CNS infections, autoimmune and metabolic disorders and mitochondriopathies was negative. He was discharged on hospital day seven after further improvement in his condition.

At a follow-up evaluation after six weeks, his family said that he had recovered completely. Examination revealed only minor neurological and behavioral deficits. His mental status examination was normal.

A detailed history about exposure to toxins was obtained from the patient. He sold solvents to clean metal machinary. When the companies to which he sold supplies had a surplus, he would take the extras from them and place them in the rear of his car. A complete review of the items in his car revealed several products that contained toluene that had been there for at least six to eight months. He also recalled a strong chemical smell in his car. His family corroborated the fact that "his car smelled". He also recalled having intermittent problems with concentration and balance for months.

A repeat MRI brain at follow-up showed a reduction in the white matter changes although they still persisted bilaterally in the periventricular region (Figure 3 and 4). Since the other clinical tests and investigations failed to detect a cause for the patient's condition, we concluded that he developed leukoencephalopathy from a persistent accidental exposure to toluene. He and his family members denied that he had purposefully inhaled toluene and there were no sources of toluene in his house to abuse.

Organic solvent abuse is the most common form of toluene exposure and the abusers have reported a euphoric state similar to alcohol. ${ }^{1}$ Long-term exposure to toluene, however, can produce serious neuropsychological impairment. Neurobehavioral deficits resulting from occupational and accidental exposure to toluene have rarely been reported. ${ }^{2}$ Acute toluene exposure can lead to disorientation, drowsiness and ataxia. Chronic exposure can produce inattention, memory deficits and visuospatial impairment, which may be accompanied by neuroimaging changes.

Neuroimaging findings are usually characterized by hyperintensities in peri-ventricular, subcortical (basal ganglia, thalamus etc.) and white matter regions. ${ }^{3}$ They are thought to reflect demyelination. It has been suggested that neurotoxicity due to toluene depends upon its presence in blood and usually the neuropsychological impairment and neuroimaging findings reverse once the insult has been removed. ${ }^{4}$ This may explain the gradual clinical improvement in our patient once the exposure to toluene was eliminated. His clinical improvement was surprising considering the substantial burden of hyperintensities that remained on his Fluid Attenuated Inversion Recovery images.

This patient's presentation appears to be unique in having a neurobehavioral syndrome caused by unintentional exposure to toluene.

\section{REFERENCES}

1. Sharp CW, Rosenberg NL. Inhalants. In: Lowinson JH, Ruiz P, Millman RB, Langrod JG, editors. Substance abuse. A comprehensive textbook. Baltimore: Williams and Wilkins; 1997. p. 246-64.

2. LoSasso GL, Rapport LJ, Axelrod BN. Neuropsychological symptoms associated with low-level exposure to solvents and (meth)acrylates among nail technicians. Neuropsychiatry Neuropsychol Behav Neurol. 2001;14(3):183-9.

3. Yücel M, Takagi M, Walterfang M, Lubman DI. Toluene misuse and long-term harms: a systematic review of the neuropsychological and neuroimaging literature. Neurosci Biobehav Rev. 2008;32(5):910-26.

4. Deschamps D, Géraud C, Dally S. Cognitive functions in workers exposed to toluene: evaluation at least 48 hours after removal from exposure. Int Arch Occup Environ Health. 2001;74(4): 285-8.
From the Department of Neurology (SUQ, AJ, PES), Baylor College of Medicine, Houston; San Antonio Medical Center (ARB), San Antonio, Texas, USA.

Received January 5, 2009. Final Revisions Submitted January 14, 2009. Correspondence to: Paul E Schulz, Mail Stop: NB302, Department of Neurology, Baylor College of Medicine, 6501 Fannin Street, Houston, Texas, 77030, USA. 

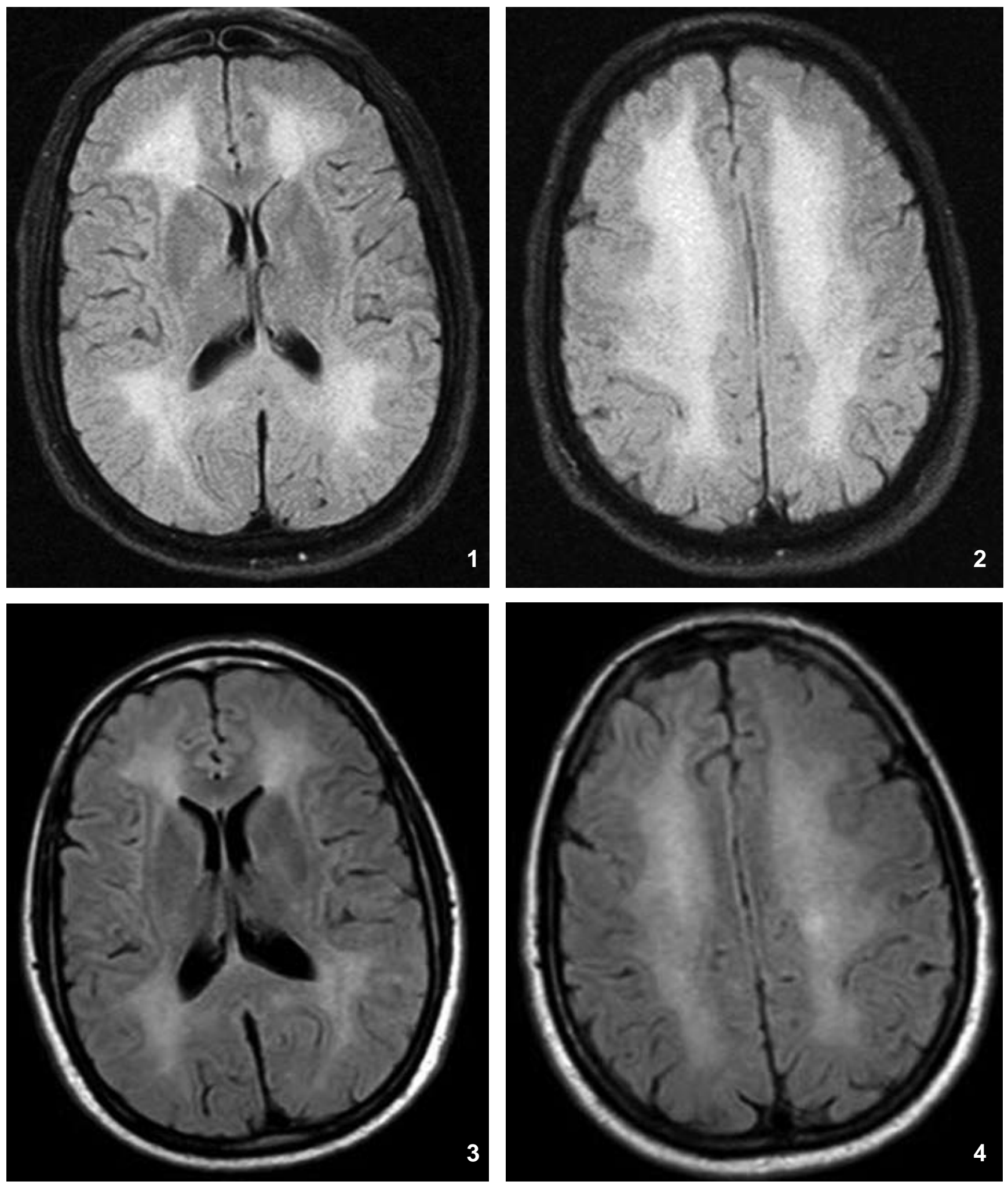

Figures: Figure (1) and (2), FLAIR images at presentation demonstrating confluent signal hyperintensities in the hemispheric white matter. (3) and (4), FLAIR images six weeks later at a time when the patient was behaviorally and cognitively normal demonstrating some improvement of the white matter changes. 